\title{
The Impact of under-Staffing of Nurses on Sedative/Analgesic Agents Administration in Pediatric Intensive Care Unit [PICU]
}

\author{
Khaled Abdelmagid, Kramer Bree, Heard Christopher \\ Department of Pediatrics, Pediatric Critical Care, University of Buffalo Oishei Children's' Hospital, Buffalo, USA \\ Email address: \\ Khaledshams1982@yahoo.com (K. Abdelmagid),bkramer@upa.chob.edu (K. Bree), cheard@upa.chob.edu(H. Christopher)
}

\section{To cite this article:}

Khaled Abdelmagid, Kramer Bree, Heard Christopher. The Impact of under-Staffing of Nurses on Sedative/Analgesic Agents Administration in Pediatric Intensive Care Unit [PICU]. Pharmaceutical Science and Technology. Vol. 5, No. 1, 2021, pp. 7-13.

doi: $10.11648 /$ j.pst.20210501.12

Received: November 18, 2020; Accepted: December 17, 2020; Published: May 21, 2021

\begin{abstract}
Institution: Department of Pediatrics, pediatric critical care, University of Buffalo Oishei Children's' hospital. Objective: To evaluate the impact of understaffing on administration of sedatives and analgesics to patients in pediatric intensive care unit [PICU]. Design: Retrospective cohort. Setting: PICU in a university-affiliated children's hospital. Patients: Fifteen intubated children 0-21 years of age admitted to the PICU in September and October 2017 at the Women's and Children's Hospital of Buffalo. Twenty-one intubated children admitted in January and February 2018 at Oishei Children's Hospital. All intubated patients in our study received sedation and analgesic drips and/or boluses. Intervention: None. Results: We used NEMS [Nine Equivalent of nursing use Manpower score] as a way of evaluating the 'appropriateness' of nurse-patient ratio. Appropriate shifts were defined as shifts with Nurse/Patients [N/P] ratio similar to that determined per NEMS. Under-staffed Shifts were shifts with N/P ratio less than determined per NEMS. Sedation burden was defined as extradoses of sedation given or rate change of drip per shift. There were no differences in age, sex, race, weight and PRISM score between patients admitted in both hospitals. There was no significant difference in sedation burden between appropriate shifts 207/429 [48.2\%] vs under-staffed shifts 26/44 [59.1\%], p-value=0.17. Linear regression was used to account for severity of disease using PRISM score, there was no significant difference between the two groups. There was significant sedation burden in shifts with higher N/P ratio [1:1 or 2:1] vs lower N/P ratio [1:2] [112/191 58.6\% vs $121 / 28143 \%, p$-value $=0.0009]$. Conclusion: We could not prove that understaffing would be associated with increase sedation. In our study group, there was increase sedation administration to patients when they had more nurses at their bedside. We think having an established sedation score and collecting these scores with the amount of sedation given during appropriate and under-staffed shifts might help controlling for some of the variables and give a more objective method to judge the patients' depth of sedation. More studies need to be conducted on the effect of nurse staffing and the amount of sedation.
\end{abstract}

Keywords: Sedation, Analgesics, NEMS, Under-staffing

\section{Introduction}

The impact of ancillary staffing ratios on provider work load and patient morbidity and mortality in critical care has been well-established. In recent studies in adults both in Asia, Europe, and the United States, the risk of death was associated with increased patient-physician and patientnurses' ratios [1, 18, 19]. Per the American Association of Colleges of Nursing, the US is projected to experience a shortage of registered nurses [RNs] as Baby Boomers age and the need for health care grows [4]. Adverse outcomes most frequently associated with decreased nursing staff include increased infection rates, postoperative complications and unplanned extubations [2, 8, 18, 19].

An important aspect in taking care of critically ill pediatric patients is administration of sedative agents. Previous studies have shown that both over-sedation and under-sedation are associated with multiple side effects, 
including inadequate pain/sedation, iatrogenic withdrawal syndrome, unplanned endotracheal tube [ETT] extubation [UPE], post-extubation stridor, extubation failure, unplanned removal of any invasive lines or tubes, ventilator associated pneumonia [VAP], catheter associated bloodstream infection [CA-BSI] and immobilization complications such as muscle wasting and pressure ulcers [10-13]. The RESTORE study [Randomized Evaluation of Sedation Titration for Respiratory failure] showed that nurse-implemented, goal directed sedation protocol did not reduce sedation-related adverse events, yet there was no discussion or comparison of the staffing, manpower or workload between intervention and control group [11]. A recent survey for hospitals in the US, showed that $94 \%$ of units have a sedation assessment tool and $86 \%$ utilize a sedation protocol which was $20 \%$ increase from previous survey done in $2005[15,16]$. Various strategies to promote light sedation is recommended in recent guidelines, as deep sedation is associated with suboptimum patient outcomes. Yet challenges emerge that decrease the adherence to these protocols. A recent survey among Scottish ICUs nurses to examine challenges against following sedation protocols, showed that three main themes: (1) recent shift in sedation practice (2) uncertainty in decision-making (3) system-level factors including ICU environment, organizational factors, and educational gaps [17]. Another survey in the US showed that nurses' attitudes toward the effectiveness of sedation in relieving patients' distress during mechanical ventilation correlated positively with their intention to administer sedatives. [15]. Few studies investigated nursing's impact on pediatric outcome and it mostly focused on the level of education and experience [20].

To our knowledge there has been no study to examine the effect of under-staffing on sedation utilization and needs in pediatric critical care.

Primary Hypothesis

We hypothesize that during the under-staffed shifts, the patients received more sedation compared to appropriatelystaffed shifts.

Secondary Hypothesis

We hypothesize that our charge nurses staffing is as appropriate as the NEMS staffing.

\section{Methods}

This retrospective cohort study was approved by our Institutional Review Board with waiver of patient consent. Our unit is a 20-bed mixed medical and surgical tertiarycare unit. Data were obtained by chart review of all intubated patients admitted to our pediatric intensive care unit [PICU] in September and October 2017 at the Women's and Children's Hospital of Buffalo. At that time our unit consisted of both closed and open rooms. Open rooms were divided by curtains and closed rooms were divided by walls with the ability to close the door to the room. We also repeated this chart review process for all intubated patients admitted to our new facility during
January and February 2018 [Oishei Children's Hospital]. At the new hospital, the PICU consists of 20 closed rooms. Our unit was moved to the new facility in November 2017. We excluded patients requiring sedation for procedures other than endotracheal intubation. This excluded patients who required sedation medications being used to treat seizures. It also excluded patients who received these medications for increased agitation secondary to ingestion [intentional or accidental]; the caveat being the inclusion of patients who were intubated and receiving mechanical ventilation as a direct result of their ingestion. We also excluded patients on sedatives for more than 2 weeks prior to initiation of the study. And finally, patients on chronic use of sedatives for at least 1 month prior to admission, were also excluded in attempt to eliminate tolerance as an influencing factor. Once the endotracheal tube was removed, the study was completed.

In our institution, the nurse-patient ratio $[\mathrm{N} / \mathrm{P}]$ is determined by the charge nurse responsible for each shift. They rely on the unit census, acuity of patient and staff available for assignment. We do not have a written protocol for N/P assignment. The assignments depend entirely on the charge nurses' experience and knowledge of the staff capabilities. The calendar day is divided into 2 shifts, each being 12 hours. Morning shift is from $7 \mathrm{am}$ to $7 \mathrm{pm}$ and the Night shift is from $7 \mathrm{pm}$ to $7 \mathrm{am}$. The charge nurse determines the N/P ratio at the beginning of each shift. To determine the appropriateness of staffing we needed an objective tool to measure our actual nurse-patient staffing against.

Recent European literature demonstrated the use of a variety of different tools that nurses can use to identify the severity of patients' conditions and nursing workload. This is to ensure safety and improve quality of care, especially in pediatric settings, in which care includes not only the children but also their parents and relatives $[5,6]$. One of these tools is the Nine Equivalents of Nursing Use Manpower [NEMS] which was based on TISS-28. The Therapeutic Intervention Scoring System [TISS] was originally presented in 1974, and some changes have been made over time, its simplified version is currently the most disseminated versions [6]. The simplified Therapeutic Intervention Scoring System [TISS-28] presented in 1996, is composed of 28 items designed to measure the severity of the disease and nursing workload [6]. Prospective observational studies showed good correlation and agreement between the TISS-28 and the NEMS, enabling validation in pediatric patients [5-8]; however, it only measures care activities delegated from therapeutic intervention and does not reflect basic nursing activity [7]. NEMS include 9 aspects of patients' care filled by nurses. EURICUS-I and II database [European ICU database] described three level of care [LOC] based on NEMS. Group 1 [NEMS <21], group 2 [NEMS 21-30] and group 3 [NEMS $>30$ ], with corresponding adjusted nurse-patient 1:3, $1: 2$ and $1: 1$ [7-9]. We used NEMS as a way of evaluating the 'appropriateness' of nurse-patient ratio. The 9 aspects of patients' care included in NEMS scoring [Table 1] are: 
basic monitoring, hourly vital signs, calculation of fluid balance, intravenous medication [boluses or continuous], use of mechanical ventilation with or without PEEP and with or without muscle relaxants, supplementary ventilator care. Also included are vasoactive medication, dialysis, patient specific intervention e.g. tracheal intubation, endoscopy, emergency operation in the past 24 hours or gastric lavage and if the patient needed specific interventions outside PICU such as surgical intervention or diagnostic procedure. The data required for the NEMS score was collected for each shift for each patient in our study. The shift was considered "appropriate "if the actual nurse-patient ratio was similar to the predicted ratio determined per NEMS. The shift was considered "understaffed" if the actual nurse-patient ratio was less than that predicted per NEMS.

We also collected the patients' characteristics including: age, sex, race, and weight, length of stay in the PICU, ventilator free days in 28 days, discharge diagnosis and their admission PRISM score. We collected the number of sedation boluses given and the number of rate changes of sedative infusion during each shift through our study period.

Table 1. NEMS Components

\begin{tabular}{|c|c|}
\hline Item & Points \\
\hline Basic monitoring Hourly vital signs, regular record and calculation of fluid balance. & 9 \\
\hline Intravenous medication Bolus or continuously, NOT including vasoactive drugs. & 6 \\
\hline $\begin{array}{l}\text { Mechanical ventilatory support Any form of mechanical/assisted ventilation, with or without PEEP [e.g., continuous positive airway pressure], } \\
\text { with or without muscle relaxant. }\end{array}$ & 12 \\
\hline Supplementary ventilatory care Breathing spontaneously through endotracheal tube; supplementary oxygen any method, except if above applies. & 3 \\
\hline Single vasoactive medication Any vasoactive drug. & 7 \\
\hline Multiple vasoactive medication More than one vasoactive drug, regardless of type and dose. & 12 \\
\hline $\begin{array}{l}\text { Specific interventions in the ICU Such as endotracheal intubation, introduction of pacemaker, cardioversion, endoscopy, emergency operation in } \\
\text { the pat } 24 \mathrm{~h} \text {, gastric lavage; routine interventions such as X-ray, echocardiography, electrocardiography, dressings, introduction of venous or } \\
\text { arterial line, are NOT included. }\end{array}$ & 5 \\
\hline $\begin{array}{l}\text { Specific intervention outside the ICU Such as surgical intervention or diagnostic procedure; The intervention/procedure is related to the severity } \\
\text { of illness of the patient and makes an extra demand upon manpower effort in the ICU. }\end{array}$ & 6 \\
\hline
\end{tabular}

Descriptive analysis was used to describe baseline patients' characteristics. Chi-square was utilized to analyze data for number of shifts with sedation burden [extradoses of sedation and rate changes of sedation infusion] per shift between appropriate and under-staffed shifts. Also shifts in which nurse-patient ratio was ( 1 to 1 or 2 to 1 ) and shifts in which nurse-patient ratio was (1 to 2). The study compared Appropriate and under-staffed day and night shifts. We further analyzed the data for significant sedation burden [shifts with $\geq 2$ extradoses of sedation given or $\geq 2$ rate changes of sedation infusion rate] per shift between appropriate and under-staffed shifts. We used linear regression to adjust for difference in NEMS score between shifts to account for severity of illness.

As mentioned above we used NEMS as objective tool to measure our actual nurse-patient staffing appropriateness. So, the study group also desired to assess the effectiveness of NEMS score to correlate with the severity of illness as determined by admit PRISM scores, sicker patients would more likely have a higher NEMS score and as such a higher $\mathrm{N} / \mathrm{P}$ ratio. This was assessed using a Receiver Operator Characteristic curve [ROC] for NEMS and admit PRISM. We as Nurse: patient ratio 1:1 per NEMS was the binary variable and PRISM was the continuous variable. The study goup also examined the actual nurse-patient staffing effectiveness to correlate with severity of illness. So, another ROC was done for actual nurse-patient staffing and admit PRISM score. Here Nurse: patient ratio 1:1 per actual nursepatient staffing was the binary variable and PRISM was the continuous variable.

\section{Definitions}

Appropriate Shifts: shifts with nurse-patient ratio [N/P] similar to that determined per NEMS.

Under-staffed Shifts: shifts with nurse-patient ratio [N/P] less than that determined per NEMS.

Sedation burden: extradoses of sedation or rate change of sedation infusion per shift

Significant sedation burden: $\geq 2$ extradoses of sedation or $\geq 2$ rate changes in sedation infusion rate per shift.

\section{Results}

A total of 36 patients were enrolled in the study, 15 were included in September and October 2017 and 21 in January and February 2018 [Table 2]. The mean age in the first 2 month of the study 61 month with standard deviation SD [ \pm 63.8$]$ and in the second 2 months 50 months SD [ \pm 72.7$] \mathrm{P}$ value 0.15 . Average of weight $21.6 \mathrm{~kg} \mathrm{SD}[ \pm 18.9]$ and in the second 2 month of the study 15.5 with SD [ \pm 25.8$]$ p-value 0.339. PRISM in the first 2 month mean 7.7 with SD $[ \pm 6.6]$ and mean in the second 2 month mean 8 with SD [ \pm 4.5$]$ with P-value 0.89. Our cohort outcome shows in [Table 3] length of stay [LOS] in the first 2 month with mean 9.3 days with SD $[ \pm 4.5]$. In the second 2 month mean LOS is 21 days with $\mathrm{SD}$ [ \pm 14.3] P-value 0.224. Ventilator free days [VFD] from total of 28 days in the first 2-month mean is 17.4 with SD [ \pm 9.4$]$ and in the second 2 month mean VFD 17.4 with SD $[ \pm 9.4]$ and in the second 2 months VFD with mean 17.4 with SD [ \pm 4.7$] \mathrm{p}$-value 0.454 . Diagnosis included in our cohort 
[Table 4] showing no statistically significant between the 2 study groups with P-value 0.46 .

Table 2. Patient demography.

\begin{tabular}{llll}
\hline Items & September/October 2017 & January/February 2018 & P-value \\
\hline Number & 15 & 21 & \\
Male [\%] & 46 & 57 & 0.155 \\
Age [m] & $61[ \pm 63.8]$ & $50[ \pm 72.7]$ & 0.894 \\
Caucasian [\%] & 60 & 76 & \\
African American [\%] & 20 & 10 & 0.399 \\
Others [\%] & 20 & 14 & 0.869 \\
Weight [kg] & $21.7[ \pm 18.9]$ & $15.15[ \pm 25.8]$ & \\
PRISM & $7.7[ \pm 6.6]$ & $8[ \pm 4.5]$ & \\
\hline
\end{tabular}

* Data represented as mean $[ \pm \mathrm{SD}]$

Table 3. Diagnosis.

\begin{tabular}{llll}
\hline Items & September/October 2017 & January/February 2018 & P-value \\
\hline Respiratory [\%] [n] & $46[7]$ & $71[15]$ & 0.46 \\
CVS [\%] & 6[] & 4[] & \\
Trauma [\%] & $20[3]$ & 5[] & \\
Neuro [\%] & 8[] & 6[] & \\
Other [\%] & $20[3]$ & $14[3]$ & \\
\hline
\end{tabular}

Table 4. Patient Outcomes.

\begin{tabular}{llll}
\hline Items & September/October 2017 & January/February 2018 & P-value \\
\hline Length of stay LOS [d] & $9.3[ \pm 4.5]$ & $21[ \pm 14.3]$ & 0.224 \\
Ventilator free day [VFD] & $17.4[ \pm 9.4]$ & $17.4[ \pm 7.7]$ & 0.454 \\
\hline
\end{tabular}

* Data represented as mean $[ \pm \mathrm{SD}]$

Table 5. Appropriate VS Under-staffed Shifts and sedation burden.

\begin{tabular}{llll}
\hline Sedation burden & Appropriate shifts \%[n=429] & Under-staffed shifts \% [n=44] & P-value \\
\hline Increase sedation burden & $48.2 \%[\mathrm{n}=207]$ & $59.1 \%[\mathrm{n}=26]$ & 0.17 \\
No increase sedation burden & $51.8 \%[\mathrm{n}=222]$ & $40.9[\mathrm{n}=18]$ & \\
\hline
\end{tabular}

No significant difference between appropriate and under-staffed shifts in sedation burden.

Sedation burden: extradoses of sedation or rate change of sedative infusion.

Our study showed no statistically significant difference between appropriate and under-staffed shifts in sedation burden with P-value 0.17 [Table 5]

Even after using linear regression to adjust the NEMS score to account for the severity of the disease and the need for more sedation there was no statistically significant difference between appropriate and under-staffed shifts P-value 0.15. In our cohort group, statistically significant sedation burden in shifts with higher nurse-patients ratio "one to one or two to one" vs shifts with lower nurse-patients ratio "one to two" with Pvalue 0.0009. [Table 6] statistical difference in sedation burden between appropriate and under-staffed shifts [day and night] with P-value 0.32 for the day shifts and P-value 0.34 for the night shifts. [Table 7] There was no statistically significant between appropriate and under-staffed shifts in significant sedation burden with P-value 0.16 [Table 8].

Table 6. Nurse-patient ratio and sedation burden.

\begin{tabular}{llll}
\hline Sedation burden & One to one Two to one \% [n=191] & One to two \% [n=281] & P-value \\
\hline Increased sedation burden & $58.6 \%[\mathrm{n}=112]$ & $43 \%[\mathrm{n}=121]$ & 0.0009 \\
No increased sedation burden & $41.4 \%[\mathrm{n}=79]$ & $57 \%[\mathrm{n}=160]$ & \\
\hline
\end{tabular}

Statistically Significantly higher sedation burden in shifts with higher nurse-patient "one to one or two to one" vs shifts with "one to two" Sedation burden: extradoses of sedation or rate change of sedative infusion

Table 7. Appropriate vs Under-staffed shifts [day and night]* and sedation burden *.

\begin{tabular}{llll}
\hline Sedation burden & Increased sedation burden* & No increased sedation burden* & P-value \\
\hline Appropriate shifts $\%[n=213] *$ & $48.1 \%[n=104]$ & $51.9 \%[n=112]$ & 0.32 \\
Under-staffed shifts $\%[n=22] *$ & $59.1 \%[n=13]$ & $40.9 \%[n=9]$ & $51.6 \%[n=110]$ \\
Appropriate shifts $\%[n=213] * *$ & $48.4 \%[n=103]$ & $40.9 \%[n=9]$ & 0.34 \\
Under-staffed shifts $\%[n=22] * *$ & $59.1 \%[n=13]$ & \\
\hline
\end{tabular}

No statistical difference in sedation burden between appropriate and under-staffed shifts [day and night].

*Day Shifts: [7am-7pm] **Night Shifts [7pm-7am]

*Sedation burden: extradoses of sedation or rate change of sedative infusion. 
Table 8. Appropriate vs Under-staffed Shifts and significant sedation burden.

\begin{tabular}{lll}
\hline Significant sedation burden* & Appropriate Shifts [n=429] & Under-staffed Shifts [n=44] \\
\hline Significant increase sedation burden & $20 \%[\mathrm{n}=86]$ & $22.7 \%[\mathrm{n}=10]$ \\
No significant increase sedation burden & $80 \%[\mathrm{n}=343]$ & $77.3 \%[\mathrm{n}=34]$ \\
\hline
\end{tabular}

No statistical difference between appropriate and under-staffed shifts in significant sedation burden

*Significant sedation burden: $\geq 2$ extradoses of sedation or $\geq 2$ rate changes in sedation infusion

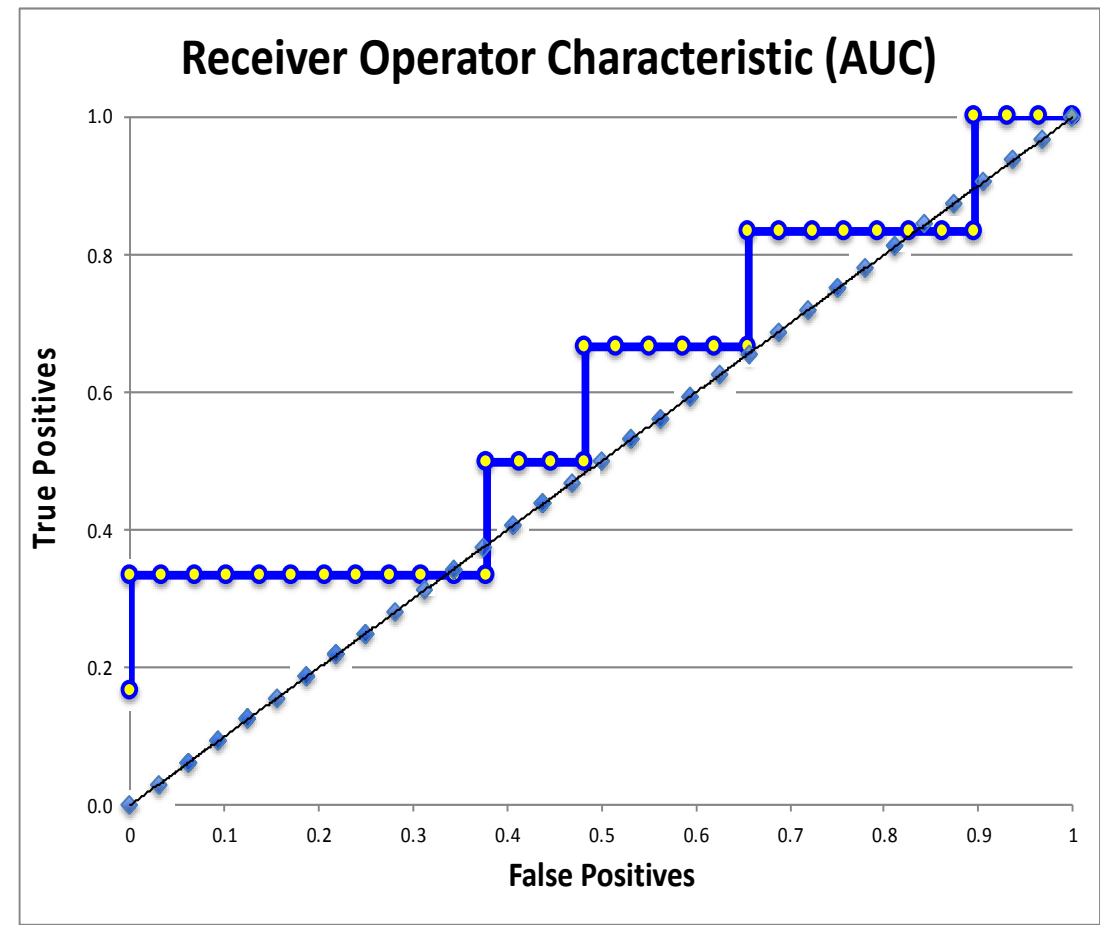

Figure 1. ROC for NEMS and admit PRISM.

ROC analysis of 1:1 [nurse/patient] ratio patients as predicted by the NEMS score matched to the admission PRISM [AUC $=0.598$ ]. poor accuracy of NEMS matching higher acuity patients with better nursing ratio.

\section{Receiver Operator Characteristic (AUC)}

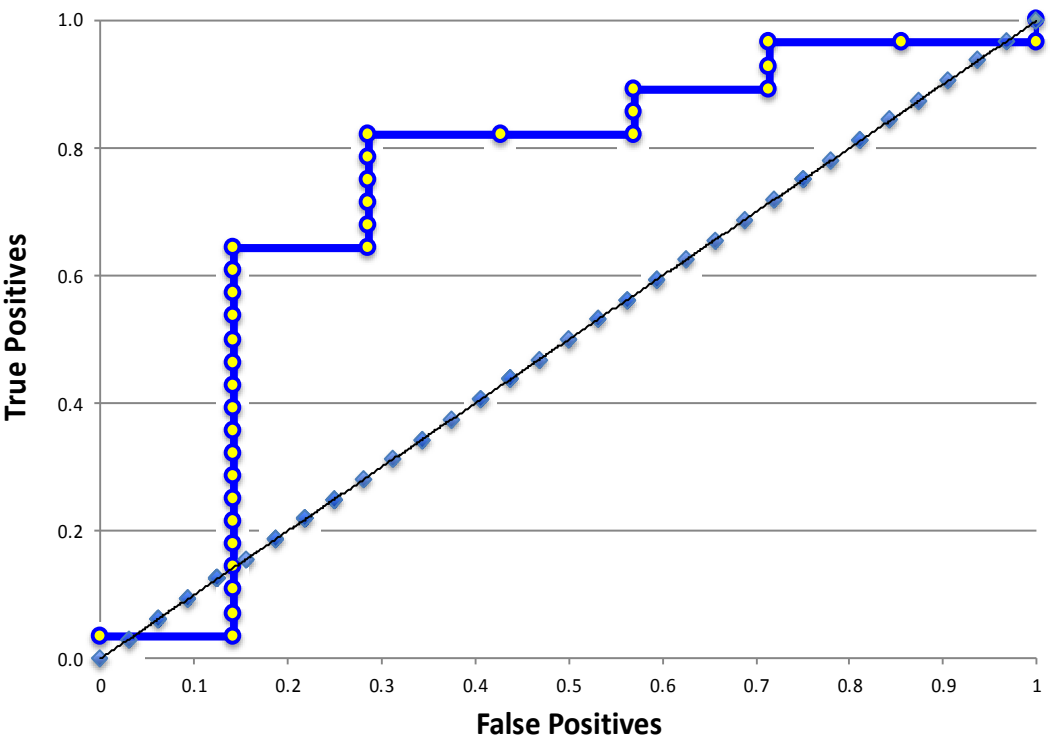

Figure 2. ROC for Actual-nurse patient ratio and admit PRISM.

ROC analysis of $1: 1$ [nurse/patient] ratio patients as chosen by the Charge nurse matched to the admission PRISM [AUC $=0.73$ ] Indicates that the charge nurse matched higher acuity patients with better nursing ratios more accurately than the NEMS score. 
ROC was done to examine the appropriateness of NEMS and actual nurse-patient staffing in capturing the severity of the disease per admit PRISM. We found that our actual nurse-patient shifts staffed [one to one] was more appropriate for the severity of the disease per the admit PRISM [AUC = 0.735] [Figure 1]. NEMS staffed shifts [one to one] not correlating as well with severity of the disease per admit PRISM [AUC $=0.598]$ [Figure 2].

\section{Discussion}

This study examined the effect of understaffing on the amount of sedation given to intubated and mechanically ventilated patients. The patients received more sedation in the shifts with higher nurse-patient staffing. As we found that there was higher sedation burden [extradoses of sedation and rate change in sedation infusion] in the shifts staffed [one to one or two to one] than shifts staffed one to two. Otherwise, there was no statistically significant difference between under-staffed and appropriately-staffed shifts in sedation burden. Even when we further examined the shifts for significant sedation burden [more than two extradoses of sedation and/or two changes in the sedative infusion] still the difference was not statistically significant. Also, understaffed day versus night shifts had no difference from the appropriately staffed shifts.

These results were against our hypothesis. It showed that patients tend to receive more sedation when they have more nurses assigned to their bedside. It is possible that these patients were sicker as well, so they needed more nursing care, and more sedatives were given. We did a linear regression to account for severity using NEMS but did not find statistical correlation.

There is a possibility that the group with less nurses who received less sedation were under-sedated. We don't rely on sedation scores to assess our patients need for sedation. We rather depend on our bedside nurses' assessment and experience especially during the different routine daily nursing activities.

We depend on our charge nurses to determine the day to day bed staffing. Our secondary hypothesis was that our charge nurses staffing is as appropriate as NEMS staffing. We have shown that our charge nurses staffing was more appropriate for higher acuity patients per admission PRISM than NEMS. Other than the initial shifts, our charge nurses staffing was similar to NEMS staffing.

The effect of understaffing was examined on multiple adult studies and few pediatric studies. Recent systemic review from Griffith et al, included fourteen studies found low nurse staffing levels were significantly associated with higher reports of missed care. [14] Also, another crosssectional survey of nurses in the National Health Service hospitals in UK by Jane et al, showed that most nurses reported that care is frequently left undone and adversely affecting quality and safety. [3] In both studies, the "missed and undone" care mentioned was most frequently: comforting or talking/ educating patients, developing/updating nursing plans and appropriate documentation. A recent survey among ICU nurses in the US showed that, although staffing levels reportedly did not influence sedation practices during mechanical ventilation for most respondents [52.6\%], 44.9\% of respondents indicated that they had sedated an intubated patient to complete other essential nursing functions [15]. In critical care, studies were consistent with findings in studies of general care population. A systemic review of literature by Penoyer et al, included 26 studies most of them suggested that decreased nurse staffing is associated with adverse outcomes in intensive care unit patient including infections, mortality, postoperative complications and unplanned extubation [2]. A multicenter observational study by Neuraz et al, showed risk of death was increased by 3.5 when patient-nurse was greater than 2.5 [1]. In pediatrics, the studies that examined the effect of understaffing on patient care are lacking. One study by Ream et al, showed that in single center pediatric critical care the likelihood of unplanned extubation was higher with higher patient-nurse ratios but also with patient acuity-nurse ratio. [13]

To our knowledge, no published study examined the effect of understaffing specifically on sedation. Despite the adverse effect of sedation on patients described in multiple studies and reviews including systemic review by Mary et al [10]. Some of the adverse events mentioned in the review: inadequate sedation and pain management, iatrogenic withdrawal syndrome, unplanned extubation and removal of any invasive tubes, extubation failure and post-extubation stridor, Ventilator associated pneumonia and pressure ulcers. In RESTORE study [Randomized Evaluation of Sedation Titration fOr Respiratory FailurE] by Curley et al, they found that the use of nurse-implemented, goal-directed sedation protocol compared with usual care did not reduce the duration of mechanical ventilation or other sedation adverse events [11]. In that study, they did not examine the effect of nurse-patient ratio on the results.

We acknowledge that our study have several limitations. This is a single center retrospective study and contains the risk of inherent biases of retrospective reviews [e.g., selection, information] which may limit generalizability. Also not all of the data may have been captured. Some of the important missing data, our charge nurses often changed nurse-patient staffing through the shifts. But the data that we gathered was never able to capture these changes that occurred during the shifts only the initial staffing.

We relied on NEMS score to evaluate the appropriateness of nurse-patient staffing. Monroy et al, reported that NEMS is simple to use and reflects the therapeutic effort of the patients admitted to a PICU; however, it only measures those cares delegated from therapeutic intervention and does not reflect the basic nursing cares [7, 8]. NEMS is used European ICU database to stratify levels of care. We examined the accuracy of NEMS to staff shifts [one to one] with the higher severity of patients determined by admit PRISM. We found 
that NEMS was less accurate than our actual nurse-patient staffing in correlating with the severity of patient. Also, our charge nurses decided the nurse-patient ratio at the beginning of the shifts. We obtained NEMS retrospectively after all the interventions was done. There was no possibility of our staffing to anticipate the possible procedures that would be done to patients. Which caused most of the early shifts deemed inappropriately staffed per NEMS. NEMS includes points for respiratory and cardiovascular support which made it inaccurate for patients who required extracorporeal support ECMO [2/36, about 5.5\%] as these supports will be provided primarily through ECMO. Another limitation was as mentioned earlier, in our unit we don't utilize any of the known sedation score to assess the depth of sedation. We generally relay on the nurses' assessment which is dependent on their expertise, training and comfort level.

\section{Conclusion}

We couldn't prove that understaffing would be associated with increase sedation. In our study group, there was increase sedation administration to patient when they had more nurses at their bedside. We believe that the group with lower nursepatient ratio was under-sedated. It is difficult to know for sure, as we do not use sedation scores in our unit. Our study did not investigate the effect of this possible under-sedation on the outcome.

We did not find NEMS score adequate for staffing of PICU as it did not correlate well with the patients' severity as determined by their admission PRISM score. Also, some acute changes occurred through shifts and can change the patient status and their staffing requirement.

We think having an established sedation score and collecting these scores with the amount of sedation given during appropriate and under-staffed shifts might help controlling for some of the variables and give a more objective method to judge the patients' depth of sedation. More studies need to be conducted on the effect of nurse staffing and the amount of sedation.

\section{References}

[1] Neuraz et al. Patient mortality is associated with staff resources and workload in the ICU: Multicenter observational study. Critical Care Medicine Aug 201543 (8): 1587.

[2] Penoyer et al. Nurse staffing and patient outcomes in critical care: a concise review.. Crit Care med Jul 2010: 38 (7): 1521.

[3] Jane Ball et al. 'Care left undone during nursing shifts: associations with workload and perceived quality of care. BMJ, quality and safety on line July 2013.
[4] Nursing shortage fact sheet. American association of colleges of nursing April 242014.

[5] Oliveria et al. Use of scores to calculate the nursing workload in a pediatric intensive care unit. Rev Bras Ter Intensiva. 2014: 26 (1): 36 .

[6] Canabarro et al. concurrent validation of nursing scores (the NEMS and TISS-28) in pediatric intensive care. Acta Paul Enferm. 2013: 26 (2): 123.

[7] Monroy et al. utilization of the NEMS in pediatric intensive care unit. Enfer Intensiva. 2002 July: 13 (3): 107.

[8] Moreno et al. Nursing staff in Intensive Care in Europe. Chest March 1998. 113 (3): 752.

[9] Miranada et al. Critical care medicine in the hospital: lessons from the EURICUS database. Med Intensiva. 2007: 31 (4): 194

[10] Defining sedation-related adverse events in the Pediatric intensive care unit. Grant et al. Heart Lung 2013; 42 (3): 171.

[11] Protocolized sedation versus usual care in pediatric patients mechanically ventilated for acute respiratory failure: RCT. RESTORE Curley et al. JAMA Jan 2015; 31 (4): 379.

[12] Oversedation from continuous sedation and extubation failures in the pediatric intensive care unit. Cole et al. Critical Car Med Dec 2013; 41.

[13] Robert S. Ream et al. Association of nursing workload and unplanned exubation in a pediatric care unit. PCCM 2007 vol. 8, No. 4. 366.

[14] Griffith et al. The association between nurse staffing and omissions in nursing care: A systematic review. Journal of Advanced nursing $2018 \mathrm{Jul} ; 74$ (7): 1474.

[15] Guttormson et al. Nurses' Attitudes and Practices Related to Sedation: A National Survey. Am J Crit Care. 2019 July; 28 (4): 255-263.

[16] Guttormson et al. Factors influencing nurse sedation practices with mechanically ventilated patients: a U.S. national survey. Intensive Crit Care Nurs. 2010; 26 (1): 44-50.

[17] Kydonaki et al. challenges and barriers to optimizing sedation intensive care: a qualitative study in eight Scottish intensive care units. BMJ open 2019; 9.

[18] Wang et al. The effect of nurse staffing on patient-safety outcomes: A cross-sectional survey. J Nurs Manag. Aug 2020; 28: $1758-1766$.

[19] Akkadechanunt et al. The Relationship Between Nurse Staffing and Patient Outcomes. The Journal of Nursing Administration Oct 2003; 33 (9): 478-85.

[20] Hickey et al. Critical Care Nursing's Impact on Pediatric patient outcomes. Ann Thoracic surg May 2016. 